Review

\title{
The emerging role of long non-coding RNAs in tumor- associated macrophages
}

\author{
Dafei Han, Yilong Fang, Yawei Guo, Wenming Hong, Jiajie Tu${ }^{\bowtie}$, Wei Wei ${ }^{\bowtie}$ \\ Institute of Clinical Pharmacology, Anhui Medical University, Key Laboratory of Anti-Inflammatory and Immune Medicine, Ministry of Education, Anhui \\ Collaborative Innovation Center of Anti-Inflammatory and Immune Medicine, Hefei, China \\ $\triangle$ Corresponding authors: Jijaie Tu, 81\# Meishan Road, Shushan Disctrict, Hefei City, Anhui Province, China, E-mail: tujiajie@ahmu.edu.cn, \\ Tel:+86055165161207, Fax: +86055165161207; Wei Wei, 81\# Meishan Road, Shushan Disctrict, Hefei City, Anhui Province, China, E-mail: wwei@ahmu.edu.cn, \\ Tel:+86055165161209, Fax: +86055165161209 \\ (C) The author(s). This is an open access article distributed under the terms of the Creative Commons Attribution License (https://creativecommons.org/licenses/by/4.0/). \\ See http:/ /ivyspring.com/terms for full terms and conditions.
}

Received: 2019.04.16; Accepted: 2019.08.24; Published: 2019.10.22

\begin{abstract}
Tumor-associated macrophages (TAMs) are an important cellular component of the tumor microenvironment (TME) and play an essential role in tumor immunity. Recently, numerous studies have indicated that long non-coding RNAs (IncRNAs) can affect several functions of TAMs. In the present review, we summarize the versatile role of IncRNAs in the polarization, epigenetic modulation, and classic signaling pathways of TAMs, which represent a potential target for tumor diagnosis or treatment.
\end{abstract}

Key words: lncRNAs, TAMs, polarization, epigenetics, NF-KB pathway

\section{Introduction}

Long non-coding RNAs (lncRNAs) are a new class of RNAs that are greater than 200 nucleotides in length and have no protein-coding ability [1]. Non-coding transcripts have been termed "junk DNA" and "transcriptional noise" in the past few decades, due to their lack of protein coding ability [2]. The number of human lncRNAs is about twice that of human protein-coding genes, and compared with the extensively studied small non-coding RNAs (microRNAs or miRNAs) and protein-coding genes, IncRNAs are still in a relatively early stage of the nomenclature, classification and identification. Growing evidence shows that IncRNAs play important roles in both physiological and pathological processes-such as the cell cycle [3], cell differentiation [4], and tumorigenesis [5-7]-and other processes, including epigenetics and chromatin remodeling $[8,9]$. A series of studies have shown that some lncRNAs are involved in tumorigenesis and cancer progression via modulating the tumor microenvironment. LncRNAs have been shown to play a role in colorectal cancer [10], hepatocellular carcinoma [11], pancreatic ductal adenocarcinoma
[12], breast cancer [13] and prostate cancer [14] etc. Therefore, elucidating the role of lncRNAs in the tumor microenvironment may help to understand the pathogenesis of tumors and reveal novel therapeutic targets.

The tumor microenvironment (TME) consists of cytokines, growth factors, and chemokines as well as a variety of immune cells induced by tumors. These gradually change during the development of cancer, which in turn promotes proliferation, invasion, angiogenesis, metastasis, immune tolerance, and drug resistance of tumor cells $[15,16]$. The immune cells in the TME mainly include $\mathrm{T}$ and $\mathrm{B}$ lymphocytes, macrophages, dendritic cells (DCs), and mast cells. Among these immune cells, the macrophages derived from the embryonic yolk sac, fetal liver, or postnatal myeloid cells are a class of immune cells are characterized by diversity and plasticity, and play an essential role in the immune response [17]. According to their polarization state of macrophages, they can be divided into classically activated macrophage (M1) and activated macrophage (M2) phenotypes. Researchers have recently realized that 
tumor-associated macrophages (TAMs) in the TME are significant to tumors. They interact with adjacent endothelial cells by releasing vascular endothelial growth factor (VEGF), which can promote angiogenesis and blood vessel anastomosis [18]. In addition, TAMs can secrete various cytokines and chemokines to recruit regulatory $\mathrm{T}$ cells into the TME, directly or indirectly inhibiting the function of $\mathrm{CD}^{+}$ and $\mathrm{CD}^{+}$effector T cells [19].

Due to the multiple roles that TAMs can play in the TME during the development of various tumors, we attempt here to summarize the function and related mechanism that lncRNAs in tumors by regulating TAMs. We will introduce the versatile role of lncRNAs in TAMs from the following perspectives: polarization, epigenetics, classic signaling pathways, and other regulatory mechanisms.

\section{The potential cellular mechanisms of interactions between lncRNAs and TAM \\ LncRNAs affect polarization of TAM}

Macrophages play an important role in innate immunity and TME. According to the polarization state, macrophages can be divided into two types, M1 and M2. M1 macrophages are induced by lipopolysaccharide (LPS) and interferon- $\gamma$ (IFN- $\gamma$ ), and have inflammatory and anti-tumor properties. Interleukin 4 (IL-4) and Interleukin 13 (IL-13) can induce M2 macrophages, which have anti-inflammatory and tumor-promoting abilities [20]. In addition, due to the plasticity of macrophages, the M1 and M2 states are not absolutely immutable. Under certain conditions, the two phenotypes can be mutually transformed. To further clarify how lncRNAs indirectly regulate tumor biology by affecting TAM polarization, several studies regarding the role played by lncRNAs in TAM polarization have been reported in recent years.

Gabrusiewicz et al. [21] demonstrated that glioblastoma patients showed a distinguished polarizatsion status of TAM. Two lncRNAs (14orf139 and SNORA25) and one lncRNA (CCDC26) were up-regulated in $\mathrm{CD}_{14}{ }^{+}$blood monocytes of glioma patients. Interestingly, in glioblastoma-infiltrating CD14 ${ }^{+}$TAM, the expression patterns of these three lncRNAs were opposite. In addition, the lncRNAs CCDC26 and RP11-419k12.1 highly expressed in M1 TAM and lncRNA RP11-26E5.1 was M2c TAM. These TAM-related lncRNAs represent potential diagnostic/treatment targets of glioma and require further investigation.

Cyclooxygenase (Cox)-2 protein plays a significant role in regulating the inflammation response in physiology and disease. LncRNA-cox-2, located approximately $50 \mathrm{~kb}$ downstream of the mouse Cox gene, is inducible in macrophages by Pam3CSK4 (an agonist of Toll-like receptor 1 and 2), LPS, or R848 (an imidazoquinoline compound with potent anti-viral and immune cell-activation properties) in a MyD88- and NF-kB-dependent manner [22]. lncRNA-cox-2 had a higher expression in M1 macrophages than non-polarized macrophages and M2 macrophages. In addition, siRNA of lncRNA-cox-2 reduced the expression levels of M1 macrophage markers, including IL-12, inducible nitric oxide synthase (iNOS), and tumor necrosis factor alpha (TNF-a) in M1 macrophages. It also increased the expression of M2 markers, such as IL-10 and arginase-1 (Arg-1), and was found in inflammatory zone 1 (Fizz-1) in M2 macrophages. Furthermore, the proliferation, migration, invasion, angiogenesis, and epithelial-mesenchymal transition (EMT) of HCC cells were repressed when co-cultured with lncRNA-cox-2 knockdown M1 macrophages. Contrastingly, lncRNA-cox-2 knockdown M2 macrophages promoted proliferation and repressed apoptosis of HCC cells. Collectively, these data indicate that lncRNA-cox-2 inhibits tumor growth and immune evasion of HCC cells by inhibiting M2 polarization in macrophages [23].

Coagulation factor $\mathrm{X}(\mathrm{FX})$ is a vitamin K-dependent plasma protein that plays an important role in regulating blood coagulation by promoting thrombin production. Studies have shown that FX is overexpressed in glioblastoma multiforme (GBM), and there is a positive correlation between FX expression and TAM density. Interestingly, FX in the TME has the ability to recruit macrophages. In addition, FX promoted M2 polarization of macrophages by increasing phosphorylation and activation of ERK1/2 and AKT in macrophages, eventually boosting GBM development. However, a lncRNA, IncRNA-CASC2c, inhibited the expression and secretion of FX, thereby inhibiting M2 polarization and tumor growth. These findings suggest that CASC2C and FX may serve as potential therapeutic targets of GBM by altering the macrophage polarization in GBM [24].

LncRNA-TUC339, which is primarily derived from HCC exosomes, is involved in the regulation of macrophage activation. Knockdown of lncRNA-TUC339 in THP-1 cells resulted in an increase in the production of pro-inflammatory cytokines and costimulatory molecules and an enhancement of the phagocytotic ability of THP-1 cells. Alternatively, lncRNA-TUC339 overexpression exerted the opposite effect. In addition, lncRNA-TUC339 is highly expressed in M2 macrophages and involved in the M2 polarization of 
macrophages. LncRNA-TUC339 expression decreased during the transition from the M2 to M1 phenotype. Overexpression of IncRNA-TUC339 in macrophages led to upregulation of M2 markers [25].

Transcribed ultra-conserved RNAs (T-UCRs) - a special class of lncRNAs that regulate mRNA transcription and translation - are a highly conserved RNA sequence between humans and mice [26]. One study found that in 30 pairs of HCC training groups and 252 pairs of HCC detection groups, the expression level of T-UCR-uc.306 in HCC cells was lower than that of adjacent tissues. There was a positive correlation between low expression of uc.306 and shorter overall survival. In addition, uc.306 was up-regulated in M1 macrophages. Target predictions indicated that beta-transducin repeat containing (BTRC) protein was a potential target of uc.306. The BTRC could interact with $\beta$-catenin (CTNNB1) in the Wnt signaling pathway. In short, macrophage-derived T-UCR uc.306 plays an important role in HCC development by regulating macrophage polarization and the Wnt signaling pathway [27].

Metastasis-associated with lung adenocarcinoma transcript-1 (MALAT1), which is overexpressed in prostate cancer (PC) tissue. PMA/IL-4-induced M2 differentiation of THP1 cells activated MALAT1 expression in PC cell lines. Knockdown of MALAT1 repressed proliferation, invasion, and tumor formation in PC cell lines. M2 macrophages secreted IL-8 could induce MALAT1 via inducing the STAT3 pathway. chromatin immunoprecipitation (ChIP) and luciferase reporter assays showed that STAT3 could bind to the MALAT1 promoter region and transcriptionally stimulate the MALAT1 expression. This report found that the IL-8/STAT3/MALAT1 pathway is function as a key regulators during prostate tumorigenesis [28].

Currently, research mainly focuses on two types of immune cells in TME: T cells and macrophages. It is generally accepted that the M1 macrophages have inflammatory and anti-tumor properties, while the M2 macrophages have anti-inflammatory and tumor-promoting abilities. LncRNAs play an important role in the development and progression of tumors, by modulating the M1 or M2 polarization of macrophages, as seen in IncRNA-cox-2, lncRNA-CASC2c, lncRNA-TUC339 and T-UCR, uc.306. These aberrantly expressed lncRNAs can be used as early diagnostic markers for certain cancers or as potential biological targets for cancer therapy (Figure 1).

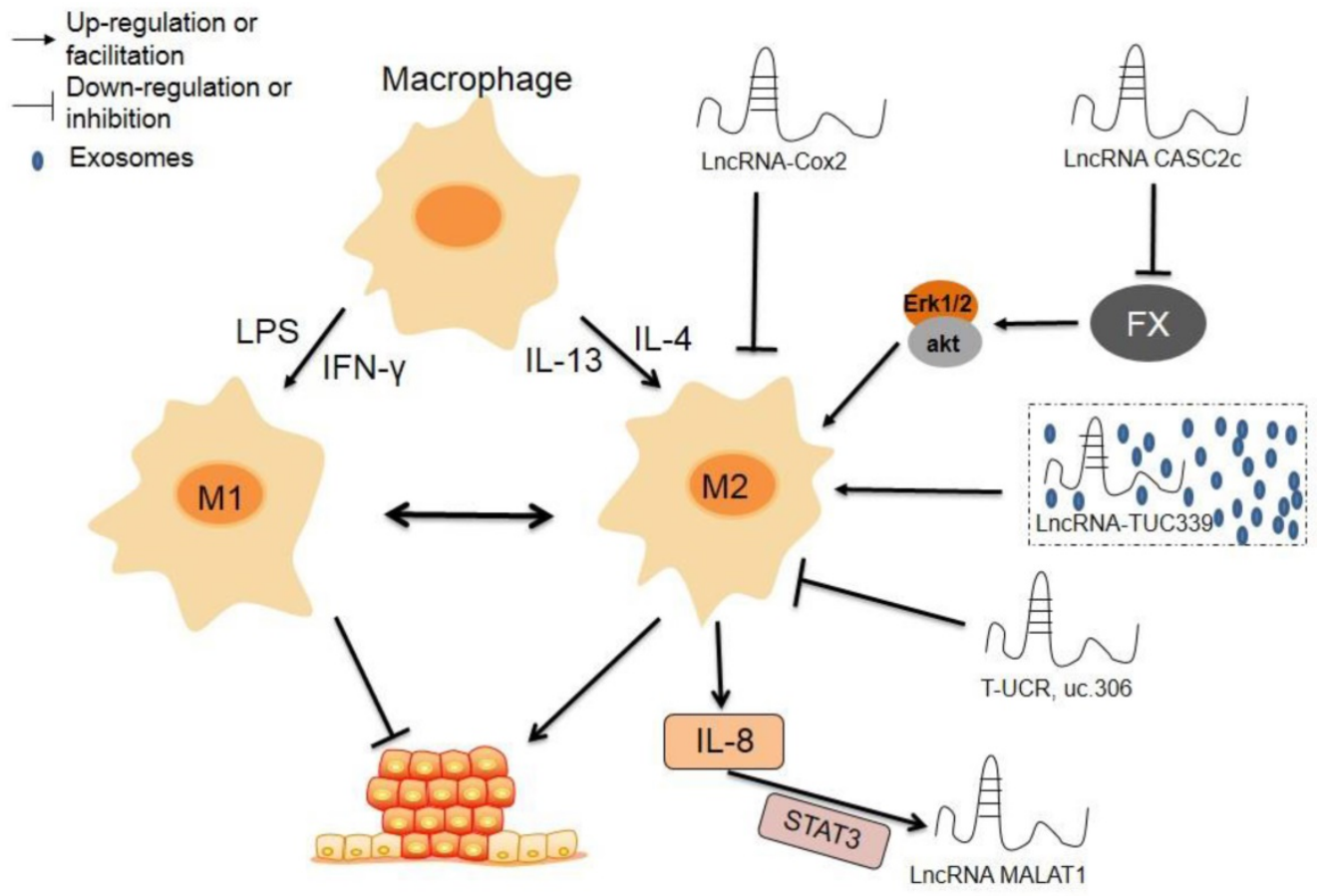

Tumor proliferation

Figure 1. The regulatory roles of IncRNAs in TAM polarization. Macrophages can transform into M1 type or M2 type induced by LPS and IFN-Y or IL-4 or IL-13, respectively, and the two phenotypes can transform mutually. LncRNAscox-2 inhibits proliferation and immune evasion of tumor by inhibiting M2 polarization in macrophages. LncRNA CASC2c suppresses the expression and secretion of FX, which lead to decrease phosphorylation and inactivation of ERK1/2 and AKT in macrophages, resulting repressed M2 polarization and decreased proliferation of tumor cells. Exosomes-derived IncRNA TUC339 promotes M2 polarization by increasing the expression of M2 markers. High expression of IncRNA T-UCR, uc.306 is associated with a higher overall survival, which may involve in promoting M1 polarization. M2 macrophages secreted IL-8 could induce the expression of MALAT1 via inducing STAT3 pathway. 


\section{The potential molecular mechanisms of interactions between IncRNAs and TAM}

\section{The interaction of IncRNAs and epigenetics affects TAMs}

Epigenetics refers to changes in gene expression levels based on non-gene sequence changes such as DNA methylation, histone modifications, chromosomal remodeling, and non-coding RNA (ncRNA) regulation. It mainly controls the function and characteristics of genes by regulating the transcription or translation processes. It also controls and maintains various cellular processes associated with basic health and disease. This section primarily describes how lncRNAs interact with epigenetic regulation in TAMs.

Colon cancer-associated transcript-1 (CCAT1) is abnormally expressed in most human cancer tissues, such as gastric cancer [29] and breast cancer [30]. Studies have shown that the levels of CCAT1 and $\mathrm{PKC \zeta}$, were quite high in M1 macrophages, while miR-148a expression was higher in TAMs. Overexpression of miR-148a promoted the expression of IL-10 and the invasive ability of tumor cells. Downregulation of CCAT1 also promoted M2 polarization of macrophages and invasion of tumor cells by increasing miR-148 expression. In addition, down-regulation of CCAT1 upregulated IL-10 production and tumor cell invasion, an effect that can be eliminated when co-transfected with miR-148a inhibitor. In addition, $\mathrm{PKC} \zeta$ was a target of miR-148a, which could exert its effect by down-regulating the expression of PKC $\zeta$. In general, downregulated CCAT1 upregulated the expression of miR-148a and then down-regulated the expression of $\mathrm{PKC} \zeta$, leading to M2 polarization of macrophages and tumor cell invasion [31].

It has been reported that NIFK-AS1 expression is quite low in TAMs isolated from endometrial cancer patients, but their miR-146a level is increased. Furthermore, overexpression of NIFK-AS1 inhibits M2 polarization (induced by IL-4) in THP-1 macrophages, leading to inhibition of proliferation, invasion, and migration of endometrial cancer cells. In addition, miR-146a interacted with NIFK-AS1. Overexpression of miR-146a attenuated the effect of NIFK-AS1 on macrophage M2 polarization and estradiol production. These findings indicate that lncRNA NIFK-AS1 inhibits M2 polarization of macrophages from the TME of endometrial cancer by targeting miR-146a [32].

LncRNA-NEAT1 (nuclear enriched abundant transcript 1 ) is a key marker in various tumors, and overexpression of NEAT1 is closely related to the occurrence and development of tumors [33-36]. Studies have found that NEAT1 and Arg-1 were highly expressed in thyroid cancer patients, and miR-214 was expressed at a basal level. In addition, NEAT1 could significantly promote the growth and metastasis of thyroid tumor cells in vitro and increase tumor size in vivo. Knockdown of NEAT1 inhibited migration, invasion, and cell survival of thyroid cancer, accompanied by decreased expression of $\beta$-catenin (a direct target of miRNA-214). Finally, NEAT1 could repress the expression of miRNA-214. Together, this evidence reveals that overexpression of NEAT1 accelerates the development of thyroid cancer and promotes the progression of thyroid cancer by regulating the expression of miRNA-214 [37].

Chemokines expressed in tumor tissues play an important role in the orientation and differentiation of macrophages by altering the microenvironment of the tumor [38, 39]. For example, CCL2 (chemokine C-C motif ligand 2) is produced primarily by a variety of tumor cells, including bladder cancer [40], and is critical for cancer metastasis [41]. LncRNA-LNMAT1 (lymph node metastasis-associated transcript 1) epigenetically activated CCL2 expression, resulting in an increased degree of trimethylation of $\mathrm{H} 3 \mathrm{~K} 4$ (H3K4me3). Upregulated CCL2 recruited macrophages to tumors, thereby accelerating lymphatics. This means that LNMAT1 promoted lymphocyte metastasis of bladder cancer and recruited macrophages via CCL2 [42].

Apart from the study of genetic mutations, the most productive research area related to tumors is that of epigenetic changes. Epigenetic modifications, without changing the DNA sequence, can control the binding of transcription factors and regulate gene expression. For example, lncRNA-CCAT1 affected the polarization state of TAMs and the invasive ability of tumor cells by regulating the expression of miR-148a. LncRNA-NIFK-AS1 targeted miR-146a, inhibited M2 polarization of macrophage endometrial carcinoma, and affected tumor cell proliferation, invasion, and migration. LncRNA-LNMAT1 epigenetically activated CCL2 expression, promoting recruitment of macrophages and metastases in the TME of bladder cancer. Reduced epigenetic changes during tumorigenesis and development - such as inhibition of DNA methylation induced by lncRNAs and repression of binding of lncRNAs to their transcription factors - lead to restoration of abnormal gene expression, providing a new avenue for research into tumor therapy based on lncRNAs (Figure 2). 


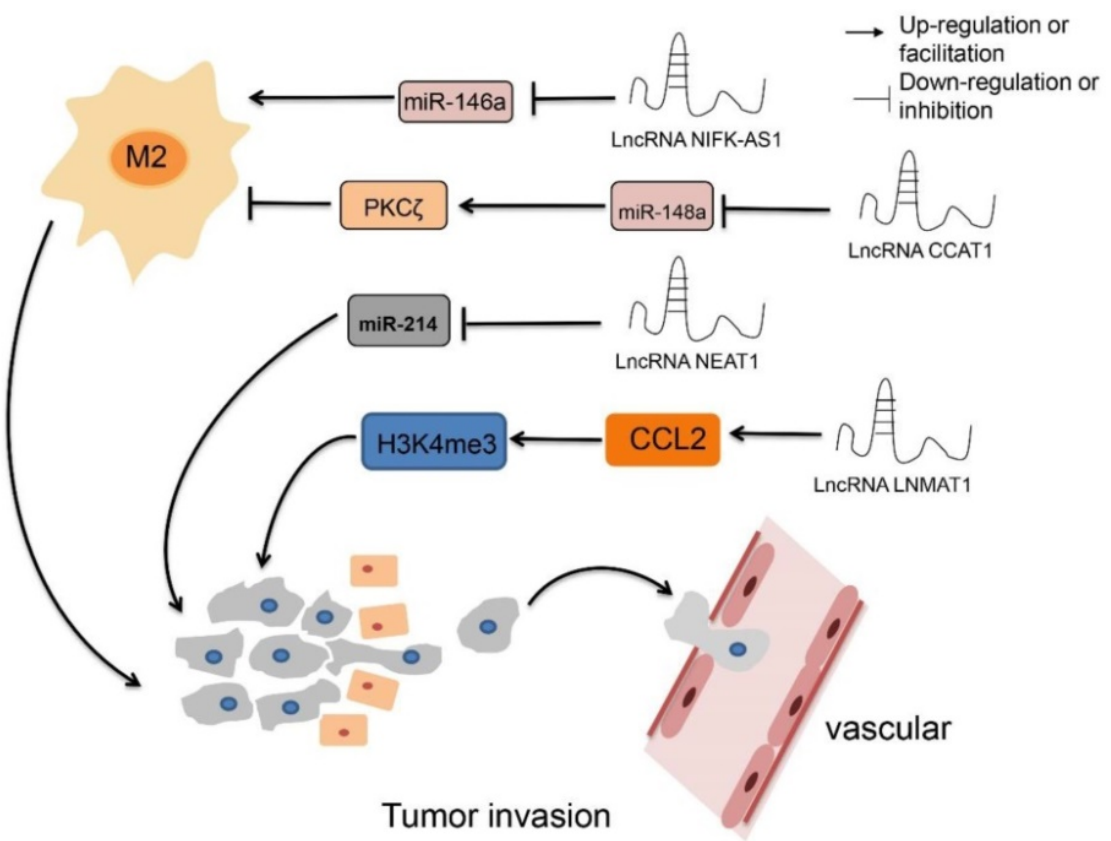

Figure 2. The regulatory roles of IncRNAs in TAM epigenetics. LncRNA CCAT1 up-regulates PKC $\zeta$ by decreasing the expression of miR-148a, resulting inhibiting M2 polarization, while down-regulate LncRNA CCAT1 have the opposite effect. Overexpression LncRNA NIFK-AS1 inhibits M2 polarization by reducing the expression level of miR-146a. LncRNA NEAT1 promotes proliferation and metastasis by inhibiting the expression of miRNA-214. LncRNA LNMAT1 promotes tumor lymphocyte metastasis and recruits macrophages through CCL2.

\section{LncRNAs regulate TAMs by affecting classic signaling pathways}

\section{The NF-KB pathway}

Nuclear factor-kB (NF-kB) has been extensively studied due to its important regulatory role in various tumors. It has been recognized that the NF-kB pathway modulates inflammatory factors and anti-apoptotic genes, thereby promoting tumorigenesis.

LncRNA-CamK-A (calcium-dependent kinase activation) was critical in the process of tumorigenesis by participating in the remodeling of the TME via activation of $\mathrm{Ca}^{2+}$-activated signaling. CamK-A activated the calcium-dependent NF-KB signaling pathway that led to tumorigenesis, angiogenesis, and macrophage recruitment. In addition, it was quite interesting to notice that inhibition of CamK-A significantly repressed tumor development in a patient-derived xenograft (PDX) model. Clinical studies have also found that CamK-A activated the NF-kB signaling pathway. Furthermore, overexpression of CamK-A is negatively correlated with the survival rate of tumor patients, suggesting that CamK-A may be a potential biomarker and a target for cancer therapy [13].

The primary TAMs were isolated from epithelial ovarian cancer (EOC) patients and exosomes were collected from primary TAM supernatants. After co-culture with HUVECs, primary TAM-derived exosomes were incorporated into HUVECs and repressed the migration of HUVECs by targeting the miR-146b-5p/TRAF6/NF-KB/MMP2 pathway. In addition, combinational treatment of TAM-derived exosomes and EOC cell line SKOV3-derived exosomes rescued the repression of HUVEC migration. Interestingly, two lncRNAs (ENST00000444164 and ENST0000043768) that from SKOV3derived exosomes were identified as NF-KB pathway-associated lncRNAs. The potential role of these two EOC exosome-derived lncRNAs is warrant of further investigation (Figure 3) [43].

\section{The AKT-P53 pathway}

TAMs are also involved in the regulation of other signaling pathways in the TME, such as the AKT signaling pathway. Investigators analyzed altered lncRNAs in breast cancer induced by conditioned medium from cultured human THP-1 macrophages, and they found that a large number of lncRNAs were changed; including lncRNA UCA (urothelial cancer associated 1). Overexpression of UCA1 was detected in human primary breast cancer. Down-regulated UCA1 inhibited AKT phosphorylation and eliminated the invasive ability of tumor cells, which is normally induced by the conditioned medium of macrophages. These results suggest that macrophage infiltration promotes breast cancer invasion by activating IncRNA-UCA1, which may be involved in activation of the AKT signaling pathway [44]. 


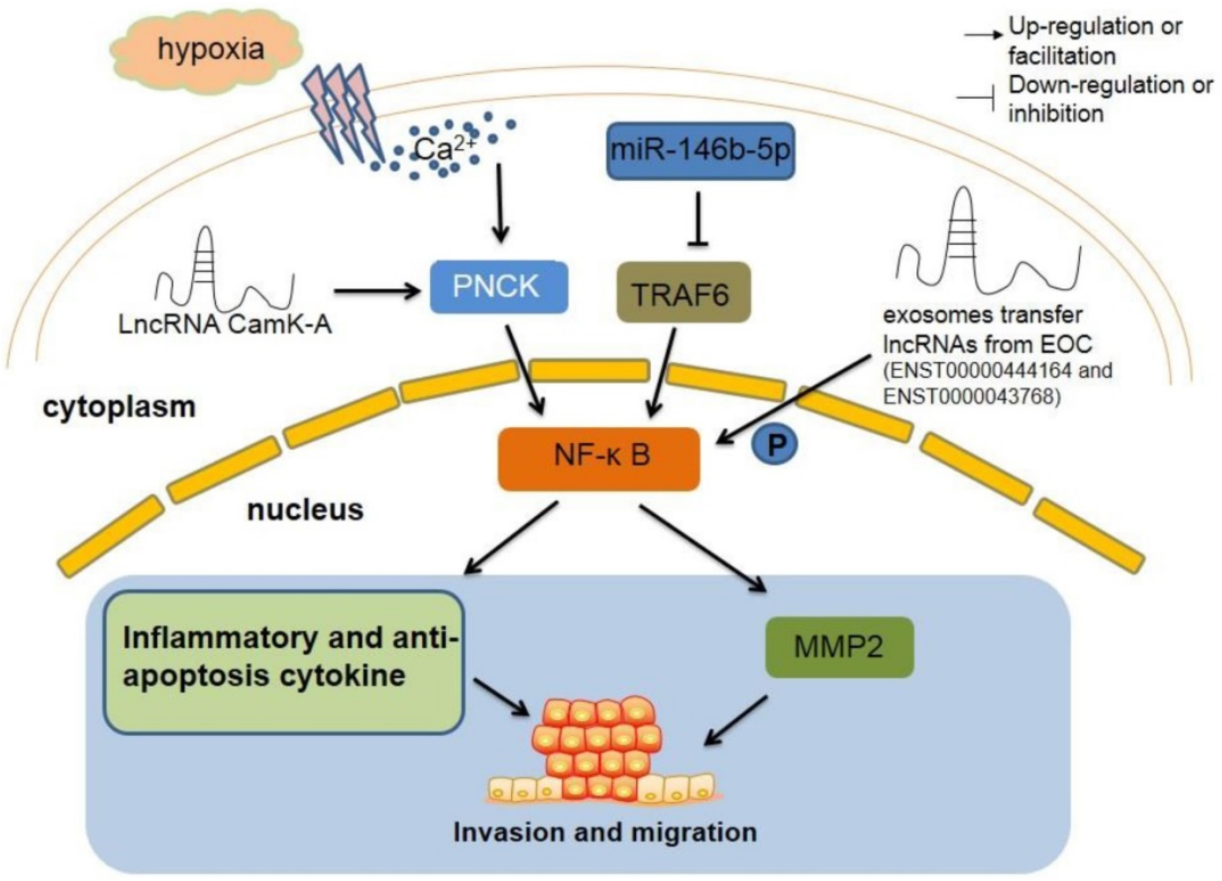

Figure 3. The regulatory roles of IncRNAs in TAM by regulating classic signaling pathway. LncRNA CamK-A motivates Ca2+-dependent NF-KB in oxygen deficiency situation, which lead to increase inflammation cytokines and macrophages recruitment, resulting tumorigenesis ultimately. TAM-derived exosomes inhibit the migration of tumor through miR-146b-5p/TRAF6/NF-KB/MMP2 signaling pathway. However, two IncRNAs from EOC exosome can reverse this suppression. NF-KB, nuclear factor-KB. PNCK, pregnancy upregulated non-ubiquitously expressed CaM kinase. TRAF6, tumor necrosis factor receptor-associated factor 6. MMP2, matrix metalloproteinase2.

LncRNA-ZFPM-AS1 (ZFPM2 antisense RNA 1) was higher in gastric tumor tissues compared to normal gastric tissues. Overexpression of ZFPM2-AS1 affected tumor size, invasion, grading, and tumor lymph node metastasis. These results were mainly linked to attenuated nuclear translocation of p53. Mechanistic studies have shown that direct binding between ZFPM2-AS1 and macrophage migration inhibitory factor (MIF) reduced the degradation of MIF, which is a potential negative regulator of p53. Knockout of MIF gene eliminated the effects of ZFPM2-AS1 on p53 expression in gastric cancer cells disappeared, indicating that ZFPM2-AS1 is involved in regulation of the ZFPM2-AS1/MIF/p53 signaling pathway. In addition, ZFPM2-AS1 protected MIF from degradation, increased MIF expression, facilitated gastric cancer proliferation, and inhibited apoptosis. These results indicate that ZFPM2-AS1 serves as an emerging oncogene in gastric cancer by protecting ZFPM2-AS1-induced MIF, providing a novel biomarker for diagnosis and a therapeutic target for gastric cancer [45].

Some classic signaling pathways, such as NF-kB, AKT and p53, etc., play important roles in the initiation and development of tumors. Aforementioned lncRNAs, such as lncRNA-CamK-A, IncRNA-UCA1, and lncRNA-ZFPM2-AS1, affect key molecules in these signaling pathways, thereby supporting tumorigenesis. Therefore, restoring these dysfunctional signaling pathways by properly regulating lncRNAs could be a potential direction for tumor therapy.

\section{LncRNAs affect TAMs through other regulatory mechanisms}

LncRNAs play important roles in the TME mainly by affecting macrophage polarization states, signaling pathways, and epigenetic alterations. They also modulate the TME through other mechanisms; for example, MALAT1 was highly expressed in thyroid tumor tissues and promoted angiogenesis of thyroid cancer by regulating expression of fibroblast growth factor 2 (FGF2) expression in TAMs [46]. Additionally, in the nutrition insufficient TME, lncRNA-JHDM1D antisense 1 (JHDM1D-AS1) was higher in pancreatic cancer cells and clinical tumor samples than in normal tissues. Overexpression of JHDM1D-AS1 promoted the growth of tumor cells both in vivo and in vitro, which was mainly related to the increased expression of some tumor-derived angiogenic factors such as human hepatocyte growth factor (hHGF), human fibroblast growth factor 1 (hFGF1), and several inflammatory response genes, including mouse matrix metalloproteinase3 (mMmp3), mouse matrix metalloproteinase9 (mMmp9), mouse S100A8 protein (mS100a8), and mouse S100A9 protein (mS100a9) [47]. Therefore, inhibiting tumor growth by inhibiting the activity of JHDM1D-AS1 is a possible strategy for effective treatment of the tumor. In addition, lncRNA Hox 
antisense intergenic RNA (HOTAIR) plays a key role in the development and metastasis of many cancers, such as hepatocellular carcinoma. Overexpression of HOTAIR upregulated the expression of C-C motif chemokine ligand 2 (CCL2), and increased the proportion of macrophages and bone marrow-derived suppressor cells (MDSCs) in peripheral blood mononuclear cells (PBMCs) [48] indicating that HOTAIR could recruit macrophages and MDSCs to the TME by elevating CCL2 (Table 1) [49].

Table 1. Other IncRNAs in tumor-associated macrophages

\begin{tabular}{|c|c|c|}
\hline LncRNAs & Description of evidence & References \\
\hline $\begin{array}{l}\text { ENST00000444164 } \\
\text { and ENST0000043768 }\end{array}$ & $\begin{array}{l}\text { two lncRNAs in EOC-derived } \\
\text { exosomes (ENST00000444164 and } \\
\text { ENST0000043768) reverse the } \\
\text { suppression of HUVEC migration by } \\
\text { TAMs in the tumor microenvironment. }\end{array}$ & {$[43]$} \\
\hline LncRNA UCA1 & $\begin{array}{l}\text { LncRNA UCA1 phosphorylated AKT } \\
\text { and facilitated invasiveness of tumor } \\
\text { cells after induced by macrophages } \\
\text { conditioned medium. }\end{array}$ & [44] \\
\hline ZFPM2-AS1 & $\begin{array}{l}\text { ZFPM2-AS1 combined to MIF and } \\
\text { reduced the degradation of MIF caused } \\
\text { nuclear translocation of p53, which } \\
\text { involved in tumor size, invasion, } \\
\text { grade, and the stage of tumor node } \\
\text { metastasis. }\end{array}$ & [45] \\
\hline LncRNA-MALAT1 & $\begin{array}{l}\text { LncRNA-MALAT1 facilitated } \\
\text { angiogenesis of thyroid cancer by } \\
\text { regulating the expression of fibroblast } \\
\text { growth factor } 2 \text { (FGF2) in TAM. }\end{array}$ & {$[46]$} \\
\hline LncRNA JHDM1D-AS1 & $\begin{array}{l}\text { LncRNA JHDM1D-AS1 increased } \\
\text { angiogenic factors derived from } \\
\text { tumor, such as, hHGF and hFGF1 and } \\
\text { inflammation-responsive genes } \\
\text { derived from host, for example, } \\
\text { mMmp3, mMmp9, mS100a8, and } \\
\text { mS100a9. }\end{array}$ & [47] \\
\hline LncRNA HOTAIR & $\begin{array}{l}\text { LncRNA HOTAIR up-regulated the } \\
\text { expression of CCL } 2 \text { and increased the } \\
\text { proportion of macrophages and } \\
\text { MDSCs in PBMCs, which associated } \\
\text { with the development and metastasis } \\
\text { of many cancers. }\end{array}$ & {$[48]$} \\
\hline
\end{tabular}

\section{Potential consequences and clinical implications of interactions between lncRNAs and TAM}

Since TAMs are a significant component of the TME, transformation of the polarization state of TAMs play an important role in the processes of tumor immunology. M1 macrophages can inhibit tumor growth, while M2 macrophages promote tumorigenesis. Therefore, it has been suggested that taking advantage of the M2 to M1 transition may represent a potential therapeutic strategy during tumor treatment. A series of studies have shown that several lncRNAs play a role in this transition, and could be considered as a potential target of TAM polarization. However, the current distinction between M1 and M2 macrophages does not fully embody the complicated characteristics of macrophages, as results indicate that some M1 type macrophage-derived cytokines (such as IL-1 $\beta$ ) could also promote tumor growth [50]. Therefore, it is essential to more accurately classify M1 and M2 macrophages both in vitro and in vivo.

\section{Future direction}

In the process of initiation and tumor development, coding genes and non-coding genes may become differentially regulated or undergo mutation in tumor tissues in order to maintain uncontrolled growth. Although some progress has been made in the study of lncRNAs, the understanding of the relationship between lncRNAs and tumors is still at a relatively early stage. Only a few lncRNAs have been well-studied in tumors and their specific structure and function remains to be fully described. Presently, studying the specific structure and function of lncRNAs in tumors is the most pressing issue. Without the proper understanding of the detailed structure and function of lncRNAs, the development of targeted lncRNA-based tumor treatments will be almost impossible. Moreover, the total number of lncRNAs in the genome is extremely large. Therefore, to identify the most essential lncRNAs in various tumors can be a much more complicated task than that of identifying the pathogenic coding gene.

In addition, to fully understand the specific function of lncRNA in TAMs, the improvement of analytical technologies for lncRNAs is also crucial. The use of advanced sequencing and gene editing technology (such as the CRISPR/Cas9 system) to achieve knock-in, knockout, and mutation, can help us to gain a greater understanding of the specific biological function of lncRNAs. However, due to the relatively long length of lncRNAs, it is difficult to investigate the tertiary structure of lncRNAs. Structure-related research into lncRNAs is an emerging field. The actions of lncRNAs largely depend on their tertiary structure. Recently, advances in high-throughput technology have made it possible to detect RNA structures in the genome. Recent developments such as parallel analysis of RNA structures (PARS), fragmentation sequencing (Frag-Seq), selective 2'-hydroxyl acylation analyzed by primer extension, and sequencing (SHAPE-Seq), and dimethyl sulfate-sequencing (DMS-Seq) have successfully begun to determine lncRNA structures across the genome. The combination of these technical methods and computational algorithms can greatly improve the accuracy of IncRNA structure prediction and validation, thereby improving our understanding 
of the structure and function of lncRNAs, accelerating the elucidation of the role of lncRNAs in tumor progression and other diseases, and enabling lncRNA-based tumor therapies.

\section{Abbreviations}

TAMs: tumor-associated macrophages; TME: the tumor microenvironment; LncRNAs: long non-coding RNAs; DCs: dendritic cells; VEGF: vascular endothelial growth factor; LPS: lipopolysaccharide;

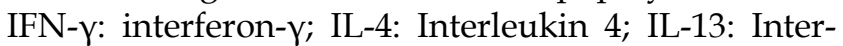
leukin 13; Cox: Cyclooxygenase; Pam3CSK4: an agonist of Toll-like receptor 1/2 (TLR1/2); R848: an imidazoquinoline compound with potent anti-viral and immune cell-activation properties; iNOS: inducible nitric oxide synthase; TNF-a: tumor necrosis factor alpha; Arg-1: arginase-1; Fizz-1: found in inflammatory zone 1; EMT: epithelialmesenchymal transition; HCC: hepatocellular carcinoma; FX: coagulation factor X; GBM: glioblastoma multiforme; ERK: extracellular signalrelated kinase; T-UCRs: transcribed ultra-conserved RNAs; BTRC: beta-transducin repeat containing; CCAT1: colon cancer-associated transcript-1; NEAT1: nuclear enriched abundant transcript 1; LNMAT1: lymph node metastasis-associated transcript 1 ; NF-kB: nuclear factor-kB; CamK-A: calcium-dependent kinase activation; PDX: patient-derived xenograft; EOC: epithelial ovarian cancer; UCA: urothelial cancer associated 1; ZFPM-AS1: ZFPM2 antisense RNA 1; MIF: migration inhibitory factor; MALAT1: metastasis-associated lung adenocarcinoma transcript 1; FGF2: fibroblast growth factor 2; JHDM1D-AS1: JHDM1D antisense 1; hHGF: human hepatocyte growth factor; hFGF1: human fibroblast growth factor 1; mMmp3: mouse matrix metalloproteinase3; mMmp9: mouse matrix metalloproteinase9; mS100a8: mouse S100A8 protein; mS100a9: mouse S100A9 protein; HOTAIR: hox antisense intergenic RNA; MDSCs: marrow-derived suppressor cells; PBMCs: peripheral blood mononuclear cells; PARS: parallel analysis of RNA structures; Frag-Seq: fragmentation sequencing; SHAPE-Seq: selective 2'-hydroxyl acylation analyzed by primer extension, and sequencing; DMS-Seq: dimethyl sulfate-sequencing.

\section{Acknowledgement}

This study was supported by the National Natural Science Foundation of China (81673444), Natural Science Foundation of Anhui Province for young scholars (1708085QH200) and Grants for Scientific Research of BSKY from Anhui Medical University (4501041101).

\section{Competing Interests}

The authors have declared that no competing interest exists.

\section{References}

1. Iyer MK, Niknafs YS, Malik R, et al. The landscape of long noncoding RNAs in the human transcriptome. Nat Genet. 2015; 47: 199-208.

2. Wright MW, Bruford EA. Naming 'junk': human non-protein coding RNA (ncRNA) gene nomenclature. Hum Genomics. 2011; 5: 90-8.

3. $\mathrm{Hu}$ YW, Kang CM, Zhao JJ, et al. LncRNA PLAC2 down-regulates RPL36 expression and blocks cell cycle progression in glioma through a mechanism involving STAT1. J Cell Mol Med. 2018; 22: 497-510.

4. Guo W, Lei W, Yu D, et al. Involvement of lncRNA-1700040D17Rik in Th17 cell differentiation and the pathogenesis of EAE. Int Immunopharmacol. 2017; 47: 141-49.

5. Xiao J, Lv Y, Jin F, et al. LncRNA HANR Promotes Tumorigenesis and Increase of Chemoresistance in Hepatocellular Carcinoma. Cell Physiol Biochem. 2017; 43: 1926-38.

6. Wu XS, Wang F, Li HF, et al. LncRNA-PAGBC acts as a microRNA sponge and promotes gallbladder tumorigenesis. EMBO Rep. 2017; 18: 1837-53.

7. Zhou S, Yu L, Xiong M, Dai G. LncRNA SNHG12 promotes tumorigenesis and metastasis in osteosarcoma by upregulating Notch2 by sponging miR-195-5p. Biochem Biophys Res Commun. 2018; 495: 1822-32.

8. Cao X, Xu J, Yue D. LncRNA-SNHG16 predicts poor prognosis and promotes tumor proliferation through epigenetically silencing p21 in bladder cancer. Cancer Gene Ther. 2018; 25: 10-17.

9. Postepska-Igielska A, Giwojna A, Gasri-Plotnitsky L, et al. LncRNA Khps1 Regulates Expression of the Proto-oncogene SPHK1 via Triplex-Mediated Changes in Chromatin Structure. Mol Cell. 2015; 60: 626-36.

10. Wu K, Zhao Z, Liu K, Zhang J, Li G, Wang L. Long noncoding RNA lnc-sox5 modulates CRC tumorigenesis by unbalancing tumor microenvironment. Cell Cycle. 2017; 16: 1295-301.

11. Zheng X, Zhang Y, Liu Y, et al. HIF-2alpha activated IncRNA NEAT1 promotes hepatocellular carcinoma cell invasion and metastasis by affecting the epithelial-mesenchymal transition. J Cell Biochem. 2018; 119: 3247-56.

12. Sun YW, Chen YF, Li J, et al. A novel long non-coding RNA ENST00000480739 suppresses tumour cell invasion by regulating OS-9 and HIF-1alpha in pancreatic ductal adenocarcinoma. Br J Cancer. 2014; 111: 2131-41.

13. Sang LJ, Ju HQ, Liu GP, et al. LncRNA CamK-A Regulates $\mathrm{Ca}(2+)$-Signaling-Mediated Tumor Microenvironment Remodeling. Mol Cell. 2018; 72: 71-83.e7.

14. Zhu $M$, Chen Q, Liu X, et al. IncRNA H19/miR-675 axis represses prostate cancer metastasis by targeting TGFBI. Febs j. 2014; 281: 3766-75.

15. Allinen M, Beroukhim R, Cai L, et al. Molecular characterization of the tumor microenvironment in breast cancer. Cancer Cell. 2004; 6: 17-32.

16. Moroishi T, Hayashi T, Pan WW, et al. The Hippo Pathway Kinases LATS1/2 Suppress Cancer Immunity. Cell. 2016; 167: 1525-39 e17.

17. Locati M, Mantovani A, Sica A. Macrophage activation and polarization as an adaptive component of innate immunity. Adv Immunol. 2013; 120: 163-84.

18. Baer C, Squadrito ML, Iruela-Arispe ML, De Palma M. Reciprocal interactions between endothelial cells and macrophages in angiogenic vascular niches. Exp Cell Res. 2013; 319: 1626-34.

19. Adeegbe DO, Nishikawa $\mathrm{H}$. Natural and induced T regulatory cells in cancer. Front Immunol. 2013; 4: 190.

20. Zhou D, Huang C, Lin Z, et al. Macrophage polarization and function with emphasis on the evolving roles of coordinated regulation of cellular signaling pathways. Cell Signal. 2014; 26: 192-7.

21. Gabrusiewicz K, Rodriguez B, Wei J, et al. Glioblastoma-infiltrated innate immune cells resemble M0 macrophage phenotype. JCI Insight. 2016; 1: e85841.

22. Carpenter S, Aiello D, Atianand MK, et al. A long noncoding RNA mediates both activation and repression of immune response genes. Science. 2013; 341: 789-92.

23. Ye $Y, X u$ Y, Lai $Y$, et al. Long non-coding RNA cox-2 prevents immune evasion and metastasis of hepatocellular carcinoma by altering M1/M2 macrophage polarization. J Cell Biochem. 2018; 119: 2951-63.

24. Zhang Y, Feng J, Fu H, et al. Coagulation Factor X Regulated by CASC2c Recruited Macrophages and Induced M2 Polarization in Glioblastoma Multiforme. Front Immunol. 2018; 9: 1557.

25. Li X, Lei $\mathrm{Y}, \mathrm{Wu} \mathrm{M}, \mathrm{Li}$ N. Regulation of Macrophage Activation and Polarization by HCC-Derived Exosomal lncRNA TUC339. Int J Mol Sci. 2018; 19: 2958.

26. Bejerano G, Pheasant M, Makunin I, et al. Ultraconserved elements in the human genome. Science. 2004; 304: 1321-5.

27. Luo HL, Chen J, Luo T, et al. Downregulation of Macrophage-Derived T-UCR uc.306 Associates with Poor Prognosis in Hepatocellular Carcinoma. Cell Physiol Biochem. 2017; 42: 1526-39.

28. Zheng T, Ma G, Tang M, Li Z, Xu R. IL-8 Secreted from M2 Macrophages Promoted Prostate Tumorigenesis via STAT3/MALAT1 Pathway. Int J Mol Sci. 2018; 20: 98 
29. Li Y, Zhu G, Ma Y, Qu H. LncRNA CCAT1 contributes to the growth and invasion of gastric cancer via targeting miR-219-1. J Cell Biochem. 2017; [Epub ahead of print].

30. Lai Y, Chen Y, Lin Y, Ye L. Down-regulation of LncRNA CCAT1 enhances radiosensitivity via regulating $\mathrm{miR}-148 \mathrm{~b}$ in breast cancer. Cell Biol Int. 2018; 42: 227-36.

31. Liu J, Ding D, Jiang Z, Du T, Liu J, Kong Z. Long non-coding RNA CCAT1/miR-148a/PKCzeta prevents cell migration of prostate cancer by altering macrophage polarization. Prostate. 2019; 79: 105-12.

32. Zhou YX, Zhao W, Mao LW, et al. Long non-coding RNA NIFK-AS1 inhibits M2 polarization of macrophages in endometrial cancer through targeting miR-146a. Int J Biochem Cell Biol. 2018; 104: 25-33.

33. Liu C, Feng Z, Chen T, et al. Downregulation of NEAT1 reverses the radioactive iodine resistance of papillary thyroid carcinoma cell via miR-101-3p/FN1/PI3K-AKT signaling pathway. Cell Cycle. 2019; 18: 167-203.

34. Zhang H, Cai Y, Zheng L, Zhang Z, Lin X, Jiang N. Long noncoding RNA NEAT1 regulate papillary thyroid cancer progression by modulating miR-129-5p/KLK7 expression. J Cell Physiol. 2018; 233: 6638-48.

35. Liu X, Liang Y, Song R, et al. Long non-coding RNA NEAT1-modulated abnormal lipolysis via ATGL drives hepatocellular carcinoma proliferation. Mol Cancer. 2018; 17: 90.

36. Qian K, Liu G, Tang Z, et al. The long non-coding RNA NEAT1 interacted with miR-101 modulates breast cancer growth by targeting EZH2. Arch Biochem Biophys. 2017; 615: 1-9.

37. Li JH, Zhang SQ, Qiu XG, Zhang SJ, Zheng SH, Zhang DH. Long non-coding RNA NEAT1 promotes malignant progression of thyroid carcinoma by regulating miRNA-214. Int J Oncol. 2017; 50: 708-16.

38. Sokol CL, Luster AD. The chemokine system in innate immunity. Cold Spring Harb Perspect Biol. 2015; 7: a016303.

39. Halama N, Zoernig I, Berthel A, et al. Tumoral Immune Cell Exploitation in Colorectal Cancer Metastases Can Be Targeted Effectively by Anti-CCR5 Therapy in Cancer Patients. Cancer Cell. 2016; 29: 587-601.

40. Bonapace L, Coissieux MM, Wyckoff J, et al. Cessation of CCL2 inhibition accelerates breast cancer metastasis by promoting angiogenesis. Nature. 2014; 515: 130-3.

41. Zhang J, Lu Y, Pienta KJ. Multiple roles of chemokine (C-C motif) ligand 2 in promoting prostate cancer growth. J Natl Cancer Inst. 2010; 102: 522-8.

42. Chen $\mathrm{C}, \mathrm{He} \mathrm{W}$, Huang J, et al. LNMAT1 promotes lymphatic metastasis of bladder cancer via CCL2 dependent macrophage recruitment. Nat Commun. 2018; 9: 3826.

43. Wu $\mathrm{Q}, \mathrm{Wu} X$, Ying $\mathrm{X}$, et al. Suppression of endothelial cell migration by tumor associated macrophage-derived exosomes is reversed by epithelial ovarian cancer exosomal lncRNA. Cancer Cell Int. 2017; 17: 62.

44. Chen S, Shao C, Xu M, et al. Macrophage infiltration promotes invasiveness of breast cancer cells via activating long non-coding RNA UCA1. Int J Clin Exp Pathol. 2015; 8: 9052-61.

45. Kong F, Deng X, Kong X, et al. ZFPM2-AS1, a novel lncRNA, attenuates the p53 pathway and promotes gastric carcinogenesis by stabilizing MIF. Oncogene. 2018; 37: 5982-96.

46. Huang JK, Ma L, Song WH, et al. LncRNA-MALAT1 Promotes Angiogenesis of Thyroid Cancer by Modulating Tumor-Associated Macrophage FGF2 Protein Secretion. J Cell Biochem. 2017; 118: 4821-30.

47. Kondo A, Nonaka A, Shimamura T, et al. Long Noncoding RNA JHDM1D-AS1 Promotes Tumor Growth by Regulating Angiogenesis in Response to Nutrient Starvation. Mol Cell Biol. 2017; 37: e00125-17.

48. Fujisaka $\mathrm{Y}$, Iwata $\mathrm{T}$, Tamai $\mathrm{K}$, et al. Long non-coding RNA HOTAIR up-regulates chemokine (C-C motif) ligand 2 and promotes proliferation of macrophages and myeloid-derived suppressor cells in hepatocellular carcinoma cell lines. Oncol Lett. 2018; 15: 509-14.

49. Heubach J, Monsior J, Deenen R, et al. The long noncoding RNA HOTAIR has tissue and cell type-dependent effects on HOX gene expression and phenotype of urothelial cancer cells. Mol Cancer. 2015; 14: 108.

50. Feng X, Szulzewsky F, Yerevanian A, et al. Loss of CX3CR1 increases accumulation of inflammatory monocytes and promotes gliomagenesis. Oncotarget. 2015; 6: 15077-94. 